\title{
Three Steps for a Safe Pancreaticoduodenectomy: Neither Pancreatic Texture nor Duct Size Matters
}

\section{Yu-Chung Chang ${ }^{1-3 *}$}

${ }^{1}$ Department of Surgery, National Cheng Kung University Hospital, College of Medicine, National Cheng Kung University, Taiwan ${ }^{2}$ Department of Surgery, Chung Shan Medical University Hospital, College of Medicine, Chung Shan Medical University, Taiwan ${ }^{3}$ Department of Surgery, Masuda Medical Association Hospital, Japan

\begin{abstract}
Background: I have designed a Q-shape biliary diversion pancreaticoduodenectomy (PD) to prevent delayedhemorrhage in case of pancreaticojejunostomy (PJ) leakage. However, eliminating PJ leakage is the ultimate goal. A PJ anastomosis technique, irrespective of soft pancreatic texture or duct-size, has been found.
\end{abstract}

Methods: Nine PDs were included. An end-to-side anastomosed proximal jejunal loop $(30 \mathrm{~cm})$, divided by a GIA stapler at its top and reapproximated using serosal sutures, was used for a choledochojejunostomy and PJs (4 fistulation, 2 duct-to-mucosa, 1 conventional and 2 whole-thickness mattress end-to-side sutures) at each divided loop. Finally, a gastro/duodenojejunostomy was made $30 \mathrm{~cm}$ distal to this looped jejunojejunostomy.

Results: There was no mortality. One minor PJ leakage healed spontaneously. One major PJ leakage had a delayed-hemorrhage crisis salvaged by continuous normal saline irrigation to neutralize the $\mathrm{pH}$ condition for bile and pancreatic juice interaction. The clinical courses were surprisingly uneventful and without fear of leakage in two PJs using whole-thickness mattress sutures. Secure ties without tearing were possible in the soft pancreatic texture.

Conclusion: Current evolutional strategies of biliary diversion reconstruction with whole-thickness-mattress PJ sutures plus normal saline irrigation can increase the safety of a pancreaticoduodenectomy by reducing PJ leakage and preventing the activation of pancreatic juice to eliminate a lethal delayed-hemorrhage.

Keywords: Pancreaticoduodenectomy; Whipple's operation; Pylorus preserving pancreaticoduodenectomy; PPPD; Delayed hemorrhage after pancreaticoduodenectomy

\section{Introduction}

Pancreaticoduodenectomy (PD) is a complex operation with high morbidity and mortality. Because of continuous advances in technique and management, improved mortality data ranging from $0 \%$ to $8.2 \%$ have been reported in systematic [1,2] and evidence-based [3] reviews. Yet pancreaticojejunostomy (PJ) leakage rates ranging from $9 \%$ to $53 \%$ (>100 cases series) remain unacceptably high [1-8]. PJ Leakage after a PD always yields a lethal crisis because of a delayed intra-abdominal hemorrhage, which is often caused by arterial erosion around a PJ anastomotic leak after long-term exposure of the arteries to the intestinal mixture of bile and pancreatic juice. This is the major cause of death after a PD $[5,6]$. The author has designed a biliary diversion reconstruction procedure [9] to prevent the bile from activating the pancreatic juice and to minimize the lethal risk caused by PJ leakage. This procedure, however, does not solve the problem of PJ leakage.

A soft pancreatic texture [5-8] and a small pancreatic duct [8] often make the pancreas a high-risk gland; they are significant risk factors for PJ leakage. To solve this problem, the author has adapted for use with PJ anastomosis the whole-thickness mattress sutures he uses for liver resections [10]. I propose three simple ways to make PD a safer procedure for every surgeon.

\section{Materials and Methods}

Three innovative strategies were used to prevent PD leakage and leakage-induced delayed hemorrhage: (1) the reconstruction aimed to divert the bile juice from the pancreatic juice, (2) the whole-thickness mattress sutures for the PJ to reduce the chance of a tear in a soft pancreas after tying the suture or in a small pancreatic duct without an anastomosis, and (3) should a leak occur despite the first two procedures, to neutralize the $\mathrm{pH}$ of the bile and pancreatic juices to 7.0 using normal saline irrigation to inactivate their interaction.
Nine patients with periampullary lesions (Table 1) that underwent biliary diversion reconstruction (Figure 1A) after PD were included. For 7 of them, conventional end-to-side PJ anastomotic techniques were used, applied, but for the two latest patients, whole-thickness mattress sutures were used (Figure 2B).

After the diagnosis had been confirmed, a conventional PD was done to excise the lesion. A subtotal gastrectomy may or may not be done, depending upon each disease condition. Reconstruction was begun by looping the proximal jejunum into a $\mathrm{Q}$ shape and anastomosing its end to its distal portion, $30 \mathrm{~cm}$ away, in an end-toside fashion (Figure 1A) and then bringing it to the hepatic hilar area through an incision in the right mesocolon. At the top of this loop, the jejunum was divided using a GIA stapler, but the mesentery was kept intact. Each divided stump was embedded into its respective jejunum using a purse-string suture. The two stumps were then re-approximated using intermittent serosal sutures (Figure 1A). For the first 7 cases, a PJ was done on the upper $1 / 3$ of the medial side of the loop with one of the following three methods: the fistulation method (4 cases), the duct-to-mucosal anastomosis method (2 cases), or the conventional end-to-side method ( 1 case). For the latest two cases, end-to-side PJ with two layers whole-thickness mattress sutures (Figure 2B) were used. Subsequently, a choledochojejunostomy was done $3 \mathrm{~cm}$ lateral to

*Corresponding author: Dr. Yu-Chung Chang, Department of Surgery, Masuda Medical Association Hospital. 1917-2 Toodacho, Masuda, Shimane 699-3676, Japan, Tel: +81-856-223611; Fax:+81-856-220407; E-mail: changmdphd@yahoo. com

Received January 20, 2017; Accepted January 28, 2017; Published February 03, 2017

Citation: Chang YC (2017) Three Steps for a Safe Pancreaticoduodenectomy: Neither Pancreatic Texture nor Duct Size Matters. Hepatol Pancreat Sci 1: 101.

Copyright: (c) 2017 Chang YC. This is an open-access article distributed under the terms of the Creative Commons Attribution License, which permits unrestricted use, distribution, and reproduction in any medium, provided the original author and source are credited. 
Citation: Chang YC (2017) Three Steps for a Safe Pancreaticoduodenectomy: Neither Pancreatic Texture nor Duct Size Matters. Hepatol Pancreat Sci 1: 101.

Page 2 of 5

\begin{tabular}{|c|c|c|c|c|c|c|c|}
\hline Case \# & PJ model & $\begin{array}{l}\text { P-duct } \\
\text { (mm) }\end{array}$ & $\begin{array}{l}\text { P-duct } \\
\text { stent }\end{array}$ & $\begin{array}{l}\text { Pancreatic } \\
\text { texture }\end{array}$ & $\begin{array}{l}\text { Cholangitis } \\
\text { episode }\end{array}$ & Complication & N/S irrigation \\
\hline 1 & Fistulation & 3 & External & Soft & No & No & No \\
\hline 2 & Duct-mucosa & 3 & External & Soft & No & Minor SMA bleeding & No \\
\hline 3 & Duct-mucosa & 2 & External & Soft & No & Minor PJ leak & No \\
\hline 4 & Fistulation & 1 & External & Soft & No & Delay gastric emptying & No \\
\hline 5 & End-to-side (conventional) & 3 & External & Soft & Once & No & No \\
\hline 6 & Fistulation & 1 & External & Soft & Several & Subhepatic abscess & No \\
\hline 7 & Fistulation & 2 & External & Soft & Several & $\begin{array}{l}\text { Major PJ leak and massive } \\
\text { bleeding }\end{array}$ & For bleeding \\
\hline 8 & $\begin{array}{l}\text { End-to-side whole-thickness mattress } \\
\text { sutures ( } 2 \text { layers) }\end{array}$ & 3 & $\begin{array}{l}\text { Internal } \\
\text { Segmental }\end{array}$ & Soft & Several & RUQ abscess & For abscess \\
\hline 9 & $\begin{array}{c}\text { End-to-side whole-thickness mattress } \\
\text { sutures ( } 2 \text { layers) }\end{array}$ & 1 & $\begin{array}{l}\text { Internal } \\
\text { Segmental }\end{array}$ & Elastic & 2 Months & No & No \\
\hline
\end{tabular}

Table 1: Anastomotic methods, pancreatic parameters and postoperative complications of the patients.

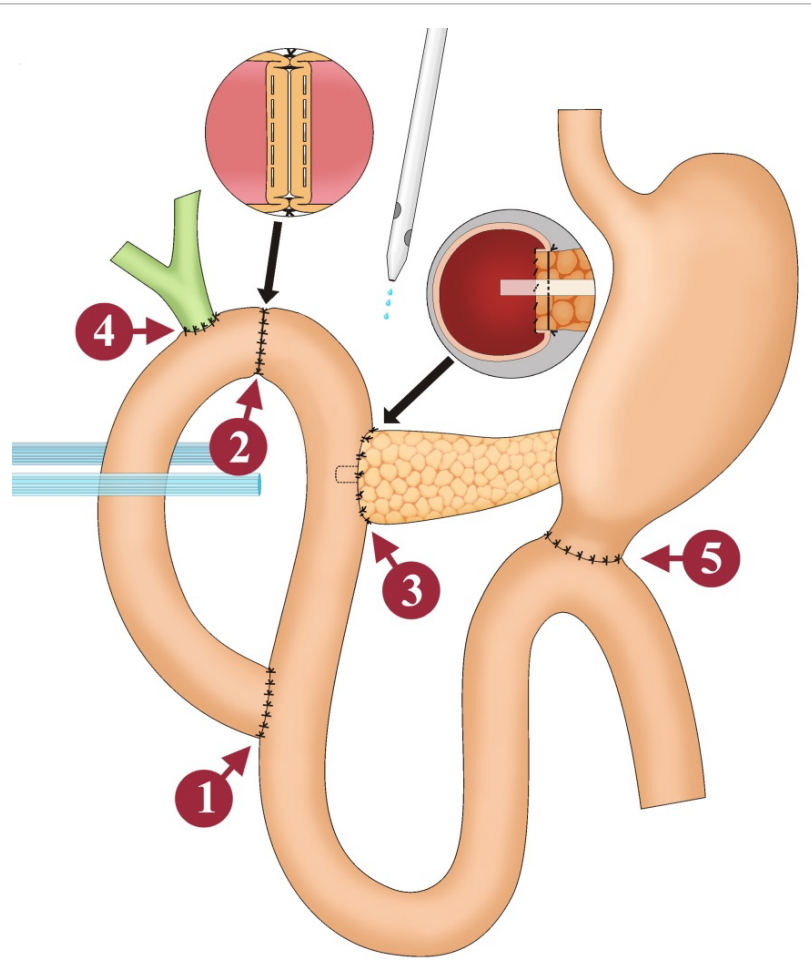

Figure 1A: A reported biliary diversion reconstruction method for pancreaticoduodenectomy, which diverts the bile flow using an independent, looped jejunal segment to prevent bile from activating pancreatic juice.

the top of the loop with a layer of intermittent 3-0 vicryl sutures. Finally, either the proximal duodenal ( 6 cases) or the gastric stump ( 3 cases) was anastomosed to the jejunum, $30 \mathrm{~cm}$ distal to the jejunojejunostomy, in an Albert-Lembert fashion. Two Penrose drains were placed separately behind and above each choledochojejunostomy and PJ for drainage and then brought out to the abdominal wall together through a right-flank stab wound. In the first 7 cases, an external stent tube matching in size was inserted into the pancreatic duct (Figure 1A) to drain the pancreatic juice. This stent tube was also brought out from the jejunum to the abdominal wall through a different stab wound with a peritonization procedure between the jejunum and the peritoneum. For the latest 2 cases, only a small segment of stent was used to identify the location of the pancreatic duct during whole-thickness mattress suture for the purpose of preventing iatrogenic ligation of the duct instead of draining the pancreatic juice. An additional Jackson-Pratt drain was placed in the

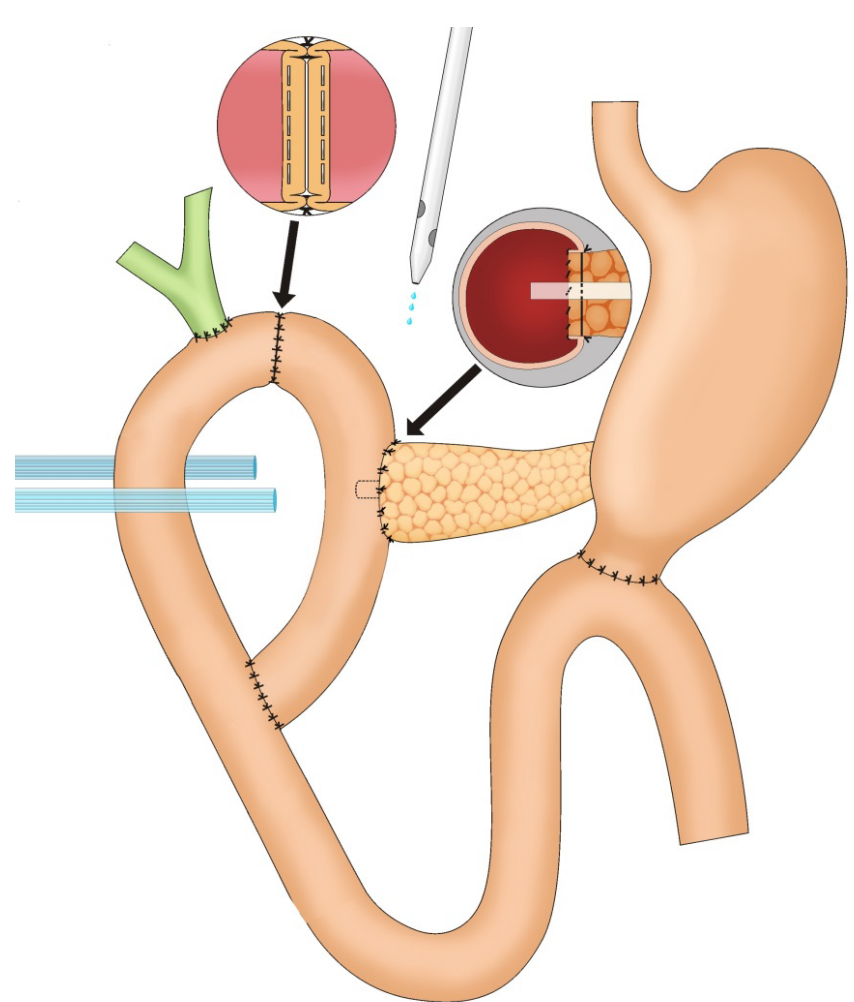

Figure 1B: A suggested modification using antiperistaltic segment of the looped jejunum for a choledochojejunostomy to reduce the complication of ascending cholangitis. Note that a normal saline irrigation catheter is placed above the PJ anastomosis in case a delayed hemorrhage occurs.

right upper quadrant (RUQ) area over the PJ anastomosis (Figure 1A) in the 9th patient, in case there was a need for normal saline irrigation.

The reconstruction procedures (Figure 1A) were as follows: 1) an end-to-side anastomosed proximal jejunal loop $(30 \mathrm{~cm}) ; 2)$ a GIA stapler division at its top and re-approximated by serosal sutures; 3 ) a choledochojejunostomy at the lateral side of the loop; 4) an end-toside PJ at the medial side of the loop (4 fistulas, 2 duct-to-mucosa, 1 conventional, and 2 whole-thickness mattress sutures; 5) finally, a gastro/duodenojejunostomy was made $30 \mathrm{~cm}$ distal to the looped jejunojejunostomy.

The one- or two-layer whole-thickness PJ pancreatic mattress suture 
1

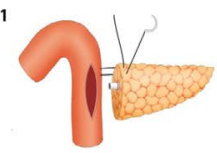

2

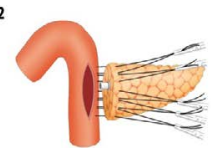

3

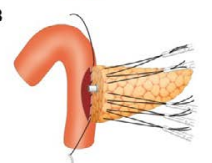

4

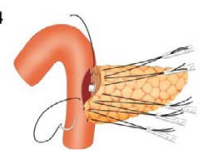

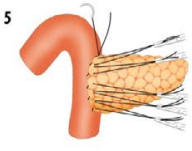

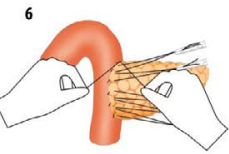

7
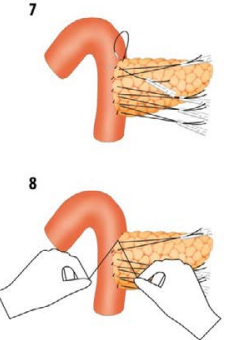
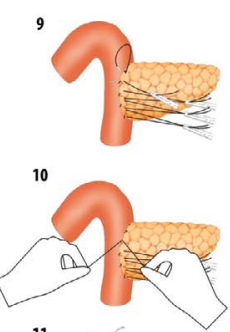

11

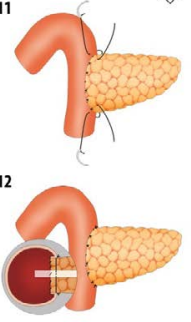

Figure 2A: Step-by-step procedures of end-to-side, one-layer whole-thickness mattress sutures to completion of PJ anastomosis with a cross-sectional view.



Figure 2B: Step-by-step procedures of end-to-side, two-layer whole-thickness mattress sutures to completion of $\mathrm{PJ}$ anastomosis with a cross-sectional view.

procedures (Figures $2 \mathrm{~A}$ and $2 \mathrm{~B}$ ) were as follows: 1) inserting a segment of pancreatic duct stent to prevent iatrogenic ligation of the duct during suturing; 2) (a) making untied whole-thickness mattress sutures by puncturing from the anterior to the posterior of the full thickness of the pancreas together with the posterior wall of the jejunum, (b) and then repuncturing backward, $8 \mathrm{~mm}$ from this site, the posterior jejunum to the anterior surface of the pancreas (3-O silks) (Figures $2 \mathrm{~A}$ and $2 \mathrm{~B}, \# 1$ ); 3 ) making either one (Figures $2 \mathrm{~A}$ and $2 \mathrm{~B}, \# 2$ ) or two rows (Figure $2 B, \# 3, \# 4$ ) of untied whole-thickness mattress sutures on both sides of the pancreatic duct; 4) making continuous sutures (3-O silk) between the anterior and posterior edges of the incised jejunum and the corresponding edges of pancreatic stump (Figure 2A \#3-\#6; 2B

\#5-\#8); 5) adding whole-layer sutures to the jejunum (anterior wall) with each of the untied sutures and then tying the mattress sutures (Figure 2A \#7-\#10; 2B \#9-\#12); finally, 6) applying two edge sutures as reinforcements (Figure 2A \#11-\#12; 2B \#13-\#15); the one-layer wholethickness sutures are completed at this stage, but stages 5 and 6 will be repeated once more for the second layer of whole-thickness sutures (Figure 2B \#16 - \#19).

\section{Results}

The clinical courses were surprisingly uneventful without fear of PJ leakage irrespective of soft pancreatic texture or small duct-size in the whole-thickness mattress suture group. Secure ties were possible without pancreatic tearing. There was no mortality. One minor pancreatic leakage (Table 1, \#2) healed spontaneously. One major leakage (Table 1, \#7) triggered by the interaction of bile and pancreatic juices after 2 weeks of constipation, had a delayed-hemorrhage crisis salvaged solely by continuous normal saline irrigation. Two pyloruspreserving patients (Table 1, \#2 and \#6) experienced a 3-week delay in gastric emptying. Two local intra-abdominal abscesses owing to poor drain placement healed after percutaneous drainage. Another patient required a reoperation for bleeding from a tiny superior mesenteric artery branch.

\section{Discussion}

The pancreatic properties of a soft parenchymal texture, small pancreatic duct, and powerful digestive capability are the Achilles heel of the PD. The inevitable problems of PJ leakage and fistulas [18] with subsequent delayed intra-abdominal bleeding remain unsolved and are still a nightmare and the major causes of postoperative morbidity and mortality. Efforts have been made to reduce PJ leakage via various types of $\mathrm{PJ}$, pancreaticogastrostomy (PG) reconstructions, and a pharmacological approach (octreotide) that decreases pancreatic secretion [1-4,7]. Nevertheless, PJ leakage and the fistula rate still remain high [1-8] even in centralized high-volume medical centers [4]. This confirms that a perfect reconstruction method has not yet been established to solve these critical issues.

\section{Solutions for a soft-textured pancreas and a small pancreatic duct: whole-thickness mattress sutures without duct anastomosis}

A pancreas with a hard texture may have less PJ leakage if conventional suturing techniques are used, but for a pancreas with a soft texture, a special technique, viz., whole-thickness mattress sutures, is required to avoid tangential shearing forces. We make wholethickness mattress sutures on liver resections using a long, straight needle $[10,11]$, which satisfactorily simplifies the difficult transection procedure and minimizes ischemic and reperfusion injury by peripheral individual inflow control [11]. We recently adapted the same concept for pancreatic sutures and discovered its merit, especially for the soft-textured pancreas: it substantially reduces the risk, presented by conventional suture techniques, of tearing the pancreas. After using whole-thickness mattress sutures, I learned that similar suture techniques had been used by Langrehr et al. [12] (from 1999, n=57, PJ fistula rate 3.5\%) and Grobmyer et al. [13] (the Blumgart anastomosis, from $2000, \mathrm{n}=187$, PJ fistula: $\mathrm{B}+\mathrm{C}$ rate $6.9 \%)$. The former incised the jejunum for telescoping or dunking the pancreatic stump (one layer only) as in the current method, but without including the incised jejunal edge inside the anastomosis. The latter used jejunal serosa to wrap the duct to the mucosal anastomosis between the jejunum and pancreas stump with mattress sutures. As to the $\mathrm{p}$-duct, the former used 
an external drainage tube, but the latter did not.

When the duct is small, the duct-to-mucosa anastomotic technique usually needs an expert hand, especially when using a microscope [14] to reduce the risk of leakage or a postoperative stricture. The easiest way to deal with the duct, no matter how large or small and no matter how good or bad one's technique, is not to suture the duct at all.

The difference between the current method and the Blumgart method [13] is to open whole layers of the jejunum to include (telescope or dunk) the pancreatic stump and duct by the dorsal and ventral walls of the incised jejunum. Instead of the one-layer of mattress sutures recommended in both the Blumgart method [13] and the Langrehr method [12], two or more rows of whole-thickness mattress sutures, including continuous marginal suturing of the pancreatic stump and incised jejunal walls, can be easily applied to offer a much more secure line of defense, if necessary, to prevent PJ anastomosis leakage. The accessory duct, if it exists, and which was not included in the Blumgart method, [13] will always be included in the PJ anastomosis method recommended by the present study. This method is so simple that it does not require expert technique, a medical center with high or low volume, a pancreas with a soft or hard texture, a specific ductal size, external p-duct stent drainage, using a microscope [14] (PJ leakage rate $11 \%$ for high risk patients [n=148]), using prophylactic octreotide or fibrin glue, duct occlusion and ligation, omental or round ligament wrapping protection or shifting a PJ to a PG.

\section{Factors causing delayed hemorrhage or pseudoaneurysm after PJ leakage: Trypsinogen, elastase or lipase}

The pathogenesis for a delayed hemorrhage or pseudoaneurysm formation is unknown; however, the digestion by trypsin and elastase of an arterial vessel wall near a PJ leak is one commonly suggested mechanism [6,15,16]. Heidenhain [17] reported in 1875 that fresh pancreatic juice has no measurable active proteases.

In 1900, Schepowalnikov discovered that pancreatic juice is activated by enterokinase. In 1939, Kunitz showed that enterokinase is an enzyme which catalyses non-active trypsinogen to its active form: trypsin. A cascade reaction then occurs, because trypsin activates pro-phospholipase and all the other proteolytic zymogens in pancreatic juice. Therefore, without enterokinase, the zymogens remain inactivated. Immunofluorescent studies using antiserum to pure human enterokinase have demonstrated its presence in man on the brush-border and glycocalyx of the enterocytes of the duodenum and proximal $10-20 \mathrm{~cm}$ of the jejunum [18].

There was only minimal mucosal enterokinase activity in the remaining proximal jejunum in 5 patients given a proximal pancreaticoduodenectomy for a periampullary tumor in an endoscopic follow-up 6 months later [18]. This finding implies that trypsin and elastase (activated by enterokinase and trypsin) [19] might not be major factors causing the delayed hemorrhage after PJ leakage following a PD in which the high-enterokinase-secreting segment of the intestine had been removed.

Skin erosion around the cutaneous enterofistula containing a mixture of bile and pancreatic juice is frequently observed clinically, but neither bile nor pancreatic leakage alone can cause such erosion. Hence, the author assumes that a fatal delayed intra-abdominal hemorrhage caused by arterial erosion around a PJ leakage is attributable to the longterm exposure of these arteries to a mixture of bile and pancreatic juice. In pancreatic enzymes, lipase (active form), activated phospholipase A2 (via trypsin), colipase, and cholesterol esterase require the cooperation of bile salts for their lipolytic action [19].

Bile diversion reconstructions, such as double Roux limb reconstruction and triple Roux on a "P" loop to separate the bile and pancreatic juice flows, may not reduce the incidence of the PJ leakage, but it results in less delayed-hemorrhage mortality [9]. Our looped reconstruction design with a disconnection in the jejunum between the choledochojejunostomy and PJ not only has the same function as the double Roux limb reconstruction in a biliary diversion, but it also preserves the mesentery structure, which gives the area better blood circulation. Because this method has only one loop, it is much simpler than the double- and triple-loop methods. Furthermore, the procedure is as safe as a PG, but without the drawback of having the pancreatic duct exposed to gastric juice.

A previously reported method [9] (Figure 1A) includes an antiperistalsis function for the $\mathrm{PJ}$ loop and aims to reduce the amount of bile reflux from the loop jejunojejunostomy to the PJ anastomosis. However, there may remain a concern of increasing ascending cholangitis on the choledochojejunostomy side; however, in the present series, this was temporary and spontaneously solved months later (the incidence was held to 20\%). Using current whole-thickness mattress sutures for PJ anastomosis eliminates the risk of PJ leakage, and the antiperistaltic loop can be adjusted to the bile diversion side (Figure 1B) to further minimize the risk of ascending cholangitis.

One minor PJ leakage, which healed spontaneously, and one major PJ leakage were encountered before applying the wholethickness mattress sutures. In the latter case, the PJ leakage occurred on postoperative day (POD) 7 , and only white pancreatic juice leakage from the drain was noticed. Biliary diversion reconstruction would have fulfilled its originally designed function of preventing the activation of pancreatic juice from the bile if the patient had not had an unexpected and undetected long-term bout of severe constipation for 14 days, which was thought to have initiated the bile reflux to the leaked PJ. Bile refluxed upward, reached the PJ, and was detected in the drain on POD 31. Oozing from the drain was first noticed on POD 39. Despite somatostatin and gabexate mesilate treatment, the bleeding was aggravated until impending shock on POD 54, when an emergency operation was arranged.

Literature on the strategy of preventing a reaction between bile and pancreatic juice is scarce. Most proteases and lipases have $\mathrm{pH}$ optima in the alkaline range [20]. While the patient was waiting for an available operating theater, we used a normal saline instillation from the fistula above the $\mathrm{PJ}$ to neutralize the patient's $\mathrm{pH}$ condition. Bleeding began to reduce gradually. Within 30 minutes, the patient's bleeding gradually slowed sufficiently to justify cancelling the scheduled reoperation. Two days later, the bleeding stopped. This patient's bleeding was massive enough to provide evidence of the efficacy of normal-saline irrigation.

Although a normal-saline irrigation after a PD has been advocated to prevent pancreatic fistulas and to reduce morbidity, [1,6,7] all three recommendations were made without mentioning theoretical or empirical supporting evidence. Using normal saline irrigation to stop a delayed massive hemorrhage after a PJ leak has never been reported.

\section{Conclusion}

In conclusion, the current concept of an end-to-side pancreatojejunostomy with whole-thickness mattress sutures and without a ductal anastomosis is a simple and easy method to solve the major technical issues of a soft pancreatic texture and a small 
Citation: Chang YC (2017) Three Steps for a Safe Pancreaticoduodenectomy: Neither Pancreatic Texture nor Duct Size Matters. Hepatol Pancreat Sci 1: 101.

pancreatic duct that cause PJ leakage. The complementary biliary diversion reconstruction diverts the bile flow using an independent, looped jejunal segment to prevent bile from activating pancreatic juice when PJ leakage is encountered. Normal saline irrigation stopped a massive delayed hemorrhage after PJ leakage in the current series. The above-mentioned three procedures will ensure a safe $\mathrm{PD}$ and make this surgery safer for more surgeons.

\section{References}

1. Li-Ling J, Irving M (2001) Somatostatin and octreotide in the prevention of postoperative pancreatic complications and the treatment of enterocutaneous pancreatic fistulas: A systematic review of randomized controlled trials. $\mathrm{Br} \mathrm{J}$ Surg 88: 190-199.

2. Poon RT, Lo SH, Fong D, Fan ST, Wong J (2002) Prevention of pancreatic anastomotic leakage after pancreaticoduodenectomy. Am J Surg 183: $42-45$

3. Martin RF, Zuberi KA (2012) The evidence for technical consideration in pancreatic resections for malignancy. Surg Clin N Am 90: 265-285.

4. Cameron JL, Riall TS, Coleman J, Belcher KA (2006) One thousand consecutive pancreaticoduodenectomies. Ann Surg 244: 10-15.

5. Yekebas EF, Wolfram L, Cataldegirmen G, Harbermann CR, Bogoevski D, et al. (2007) Postpancreatectomy hemorrhage: diagnosis and treatment: An analysis in 1669 consecutive pancreatic resections. Ann Surg 246: 269-280.

6. Fuks D, Piessen G, Huet E, Tavernier M, Zerbib P, et al. (2009) Life-threatening postoperative pancreatic fistula (grade $\mathrm{C}$ ) after pancreaticoduodenectomy: incidence, prognosis and risk factors. Am J Surg 197: 702-709.

7. Berger A, Howard TJ, Kennedy E, Sauter PK, Bower-Cherry M, et al. (2009) Does type of pancreaticojejunostomy after pancreaticoduodenectomy decrease rate of pancreatic fistula? A randomized, prospective, dual-institution trial. J Am Coll Surg 208: 738-749.

8. Muscari F, Suc B, Kirziu S, Hay JM, Fourtanier G, et al. (2005) Risk factors for mortality and intra-abdominal complications after pancreatoduodenectomy: Multivariate analysis in 300 patients. Surgery 139: 591-598.

9. Chang YC (2010) Biliary diversion Q-shape reconstruction after pancreaticoduodenectomy: Chang's reconstruction. Hepatogastroenterology 57: $945-948$.

10. Chang YC, Nagasue N, Chen CS, Lin XZ (2006) Simplified hepatic resections with the use of a Chang's needle. Ann Surg 243:169-172.

11. Chang YC, Nagasue N (2008) Blocking intrahepatic inflow and backflow using Chang's needle during hepatic resection: Chang's maneuver. HPB 10: $244-$ 248.

12. Langrehr JM, Bahra M, Jacob D, Glanemann M, Neuhaus $P$ (2005) Prospective randomized comparison between a new mattress technique and Cattell (duct to mucosa) pancreaticojejunostomy for pancreatic resection. World J Surg 29 $1111-1119$.

13. Grobmyer SR, Kooby D, Blumgart LH, Hochwald S (2010) Nove pancreaticojejunostomy with a low rate of anastomotic failure-related complications. J Am Coll Surg 210: 54-59.

14. Hashimoto $Y$, Traverso LW (2010) Pancreatic anastomotic failure rate after pancreaticoduodenectomy decreases with microsurgery. J Am Coll Surg 211: 510-521.

15. Otah E, Cushin BJ, Rozenblit GN, Neff R, Otah K, et al. (2002) Visceral artery pseudoaneurysms following pancreatoduodenectomy. Arch Surg 137: 55-59.

16. Wente MN, Veit JA, Bassi C, Dervenis C, Fingerhut A, et al. (2007) Postpancreatectomy hemorrhage (PPH): An International Study Group of Pancreatic Surgery (ISGPS) definition. Surgery 142: 20-25.

17. Heidenhain FPH (1875) Beobachtungen über die Bauchspeicheldrüse. Ber klin Wschr 198: 12

18. Bett NJ (1979) Regulation of enterokinase synthesis in animal and human small intestine by luminal signals: Its implication in upper gastrointestinal surgery. $\mathrm{Br}$ J Surg 66: 708-711.

19. Fisher WE, Anderson DK, Bell RH, Saluja AK, Brunicardi FC (2009) Pancreas. In: Schwartz's Principles of Surgery, (9 $9^{\text {th }}$ eds). Brunicardi F, Andersen D, Billiar T, et al. (eds.) McGraw Hill Professional, New York, USA. Chapter 33, pp: 1174 1175.

20. Greenberger NJ, Conwell DL, Wu BU, Banks PA (2012) Acute and chronic pancreatitis. In: Harrison's Principles of Internal Medicine, 18th Edition. Longo DL, Fauci A, Kasper D, Hauser S, Jameson JL (eds.) McGraw Hill Professional, New York, USA. Chapter 313, p: 2634. 